\title{
Feeding into old age: long-term effects of dietary fatty acid supplementation on tissue composition and life span in mice
}

\author{
Teresa G. Valencak • Thomas Ruf
}

Received: 22 April 2010/Revised: 30 September 2010/Accepted: 5 October 2010/Published online: 28 October 2010

(C) The Author(s) 2010. This article is published with open access at Springerlink.com

\begin{abstract}
Smaller mammals, such as mice, possess tissues containing more polyunsaturated fatty acids (PUFAs) than larger mammals, while at the same time live shorter lives. These relationships have been combined in the 'membrane pacemaker hypothesis of aging'. It suggests that membrane PUFA content might determine an animal's life span. PUFAs in general and certain long-chain PUFAs in particular, are highly prone to lipid peroxidation which brings about a high rate of reactive oxygen species (ROS) production. We hypothesized that dietary supplementation of either n-3 or n-6 PUFAs might affect (1) membrane phospholipid composition of heart and liver tissues and (2) life span of the animals due to the altered membrane composition, and subsequent effects on lipid peroxidation. Therefore, we kept female laboratory mice from the C57BL/6 strain on three diets (n-3 PUFA rich, n-6 PUFA rich, control) and assessed body weights, life span, heart, and liver phospholipid composition after the animals had died. We found that while membrane phospholipid composition clearly differed between feeding groups, life span was not directly affected. However, we were able to observe a positive correlation between monounsaturated fatty acids in cardiac muscle and life span.
\end{abstract}

Keywords Polyunsaturated fatty acids $\cdot$ n-3 $\cdot$ n-6 $\cdot$ Life span · Membrane composition · C57BL/6

Communicated by G. Heldmaier.

T. G. Valencak $(\bowtie) \cdot$ T. Ruf

Research Institute of Wildlife Ecology, University of Veterinary

Medicine, Savoyenstrasse 1, 1160 Vienna, Austria

e-mail: Teresa.Valencak@vetmeduni.ac.at

URL: http://www.fiwi.at/

$\begin{array}{ll}\text { Abbreviations } \\ \text { ANOVA } & \text { Analysis of variance } \\ \text { ANCOVA } & \text { Analysis of covariance } \\ \text { BMR } & \text { Basal metabolic rate } \\ \text { DHA } & \text { Docosahexaenoic acid } \\ \text { PC } & \text { Principle component } \\ \text { PUFA } & \text { Polyunsaturated fatty acid } \\ \text { PI } & \text { Peroxidation index } \\ \text { SFA } & \text { Saturated fatty acid } \\ \text { UI } & \text { Unsaturation index } \\ \text { MUFA } & \text { Monounsaturated fatty acid } \\ \text { MLSP } & \text { Maximum life span potential } \\ \text { NFE } & \text { Nitrogen free extracts } \\ \text { ROS } & \text { Reactive oxygen species }\end{array}$

\section{Introduction}

Small mammals such as mice possess tissues containing more polyunsaturated fatty acids (PUFAs) than larger mammals, while at the same time live shorter lives. This relationship has been summarised in the 'membrane pacemaker hypothesis of aging', an extension of the 'oxidative stress' hypothesis of aging which suggests that membrane fatty acid composition might determine an animal's life span (reviewed in Pamplona and Barja 2007; Hulbert et al. 2007). PUFAs are important constituents of lipid bilayers and are very prone to peroxidation, which result in extensive production of radical oxygen species (ROS) (reviewed in Hulbert et al. 2007). ROS readily interact with macromolecules (e.g., proteins and DNA), leading to tissue damage and eventually cell senescence and death as postulated by the 'free radical theory' (Brand 
2000; Barja 2004; Speakman 2005). Among PUFAs, one particular long-chain PUFA with six double bonds, docosahexaenoic acid (DHA; C22:6n-3), stands out for being especially susceptible to peroxidative damage (Turner et al. 2003; Hulbert et al. 2007). DHA belongs to the n-3 PUFA class and is eight times more prone to peroxidation than the essential fatty acid linoleic acid (C18:2) which has only two double bonds and belongs to the n- 6 PUFAs (Holman 1954). n-3 and n-6 PUFAs differ in the location of the first double bond in the molecule and thus have a variety of different biochemical properties. Importantly, due to a lack of necessary enzymes, mammals are unable to synthesise essential fatty acids such as linoleic (C18:2n-6) and linolenic acid (C18:3n-3) de novo and thus can only source them through diet. Once ingested, however, mammals can readily convert essential fatty acids to longer-chain, unsaturated fatty acid molecules.

The 'membrane pacemaker' hypothesis predicts that animal species having high contents of membrane PUFAs (in particular DHA), such as mice, live short lives because of their higher risk of lipid peroxidation (Pamplona and Barja 2007; Hulbert et al. 2007). Recently, we have suggested that the relationship between n-3 and n-6 PUFAs might determine life span in mammals, rather than the association between life span and PUFAs in general (Valencak and Ruf 2007). By statistically controlling for both body weight effects and phylogeny in a dataset of 42 mammalian species, we observed a significant negative relationship between maximum life span potential (MLSP) and the ratio between $n-3$ and n- 6 PUFAs, but not with PUFAs or DHA alone (Valencak and Ruf 2007). Together, these findings suggest a possible causal relationship between phospholipid n-3/n-6 ratios and MLSP in mammals which merits experimental investigation.

We therefore intended to influence membrane composition of our adult experimental animals, C57BL/6 mice, by supplementing their diets with $n-3$ or $n-6$ PUFAs and hence test for dietary effects on life span. Correlational evidence of a relationship between membrane fatty acid composition in three strains of mice with different life spans has previously been reported (Hulbert et al. 2006a), however, they concluded that these data could not clarify "if the relationship was one of cause and effect".

We hypothesised that if mice maintained on the n-3 rich diet lived shorter lives, the membrane pacemaker hypothesis of aging would be supported. If, on the other handmice from the control group or from the n- 6 rich diet, with lower membrane unsaturation and peroxidisability, would live equally long or even longer, we would have to reject the membrane pacemaker hypothesis. Finally, if there was any effect of high fat feeding (both experimental groups) on life span, we would expect that our control group, not being supplemented with $n-3$ or $n-6$ PUFAs would live longer than the experimental groups.

\section{Materials and methods}

Animals, husbandry, and life span study

Forty, 8-weeks-old female C57BL/6 mice, were obtained from Harlan, NL and housed in individual polycarbonate cages $(267 \times 207 \times 140 \mathrm{~mm}$, Eurostandard Type II, Tecniplast, Italy) with sawdust and paper bedding. Rodent chow (Altromin, Germany) and water were available ad libitum. The environment was regulated at $22 \pm 2{ }^{\circ} \mathrm{C}$ on a $12 \mathrm{~h}: 12 \mathrm{~h} \mathrm{~L}: \mathrm{D}$ photoperiod throughout the entire experiment. After 10 months, the animals were assigned to three experimental groups and subsequently fed the following three diets which all consisted of the rodent chow described above but either with a different oil added to the pellets or without any further supplement. Regular recordings of body weight data were started the animals already acclimatised to the diets (around day 450, see Fig. 1). The n-6 rich group $(n=12)$ received rodent chow enriched with sunflower oil (750 g per 6,000 g pellets, Osana ${ }^{\circledR}$, Austria), whereas the $\mathrm{n}-3$ rich group $(n=14)$ was kept on salmon oil (750 g per 6,000 g pellets, Perro Petfood ${ }^{\circledR}$, Austria). Both oils used did not contain any additional vitamins $C$ or E. To observe if high fat content as such, irrespective of the dominant fatty acid, would affect longevity, and to ensure that one experimental group would receive a diet with lower absolute amounts of PUFA, a control group $(n=14)$ received rodent chow without any addition of oil. The exact composition of all diets is given in Table 1. To ensure that the energy content and particularly the fat

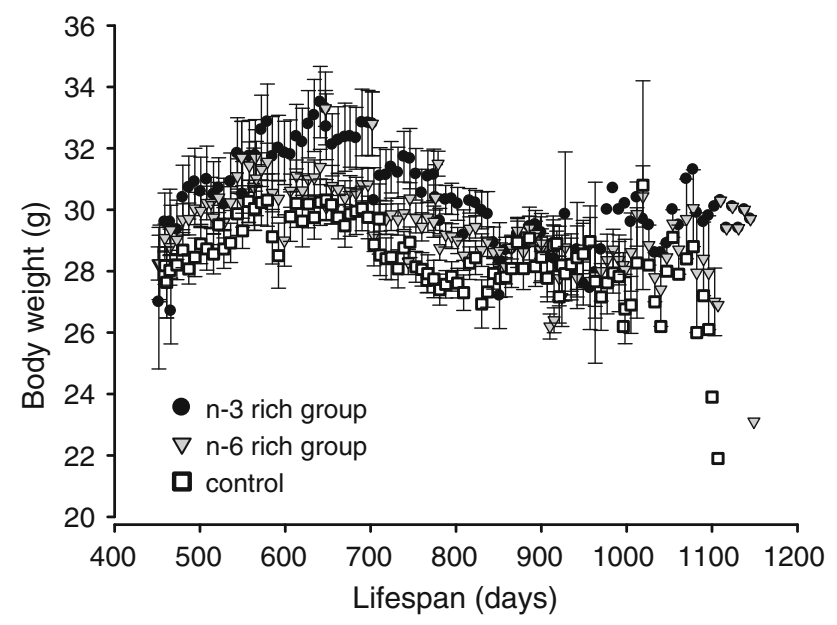

Fig. 1 Time courses of body weights of mice in the three experimental groups of mice. Mean \pm SEM, $N_{\text {total }}=40$ 
Table 1 Diet composition (wt\%) of the experimental groups

\begin{tabular}{|c|c|c|c|}
\hline & n-3 rich diet & n-6 rich diet & Control diet \\
\hline Protein $(\%)$ & 22.52 & 24.8 & 23.3 \\
\hline Fat $(\%)$ & 11.48 & 9.21 & 1.99 \\
\hline Fibre $(\%)$ & 6.04 & 4.8 & 7.8 \\
\hline NFE (\%) & 53.21 & 59.6 & 60.53 \\
\hline Energy content $\left(\mathrm{kJ} \mathrm{g}^{-1}\right)$ & 20.17 & 20.7 & 18.2 \\
\hline $\mathrm{C}: 14-0$ & 0.8 & 0.2 & 0.2 \\
\hline $\mathrm{C}: 15-0$ & 0.5 & 0.5 & 0.5 \\
\hline C:16-0 & 17.4 & 16.2 & 17.3 \\
\hline$C: 16-1 n-7$ & 0.95 & 0.15 & 0.16 \\
\hline $\mathrm{C}: 17-0$ & 1.45 & 1.42 & 1.57 \\
\hline $\mathrm{C}: 18-0$ & 2.5 & 2.5 & 2.4 \\
\hline$C: 18-1 n-9$ & 16.5 & 26.0 & 26.2 \\
\hline$C: 18-2 n-6$ & 40.5 & 50.3 & 48.5 \\
\hline$C: 18-3 n-3$ & 3.1 & 2.9 & 3.2 \\
\hline$C: 20-4 n-6$ & 0.1 & 0.01 & 0.01 \\
\hline$C: 20-5 n-3$ & 4.9 & 0.05 & 0.06 \\
\hline$C: 22-5 n-3$ & 3.4 & 0.09 & 0.07 \\
\hline$C: 22-6 n-3$ & 8.04 & 0.06 & 0.06 \\
\hline$\Sigma$ SFA & 22.65 & 20.82 & 21.97 \\
\hline$\Sigma$ MUFA & 17.45 & 26.15 & 26.36 \\
\hline$\Sigma$ PUFA & 60.04 & 53.03 & 51.67 \\
\hline$\Sigma$ N6 & 40.6 & 50.31 & 48.51 \\
\hline$\Sigma \mathrm{N} 3$ & 19.44 & 3.1 & 3.39 \\
\hline
\end{tabular}

The variability of the energy content of the diets throughout the study amounted to a coefficient of variation of 0.027

content were stable throughout the study, we analysed the dietary fat content every time the pellets were mixed with oil. The diets were chemically analysed using standardised methods for crude protein, crude fat, crude ash, and dry matter (Nehring 1960). ADF and lignin were determined by Van Soest detergent analyses (Otzelberger 1983). Fatty acid composition of the diets was assessed by transesterifying the fatty acids with boron trifluoride-methanol solution (Sigma-Aldrich, Austria) for $2 \mathrm{~h}$. For minimizing the effects of autoxidation of the fatty acids, the diets were stored in dark containers at $4{ }^{\circ} \mathrm{C}$ until they were fed to the mice and uneaten pellets were removed weekly and replaced by new ones. All animals were maintained in the same room throughout the study and no additional animals were brought into this room. The colony therefore, was effectively kept under barrier conditions. Mice were monitored daily and weighed weekly but otherwise were left undisturbed until they died naturally. If death was diagnosed, mice were weighed and examined for macroscopic pathological changes. During post-mortem dissection, we removed hearts and livers for later tissue analysis. In two cases in the control group and one case in the $n-6$ rich group, no tissues were sampled because of advanced stages of autolysis in the inner organs. If deemed unavoidable as animals were suffering, individual mice were killed following the guidelines of the Veterinary Medicine University Ethics commission and this was considered the date of death. Of all the mice that died, $30 \%$ $(n=14)$ were killed on ethical grounds, and the remainder died spontaneously. There were no significant differences in the proportions of each mode of death across the experimental groups.

\section{Lipid analysis}

Tissue samples $(0.5 \mathrm{~g}$ liver, $0.5 \mathrm{~g}$ heart $)$ were stored in plastic bags and frozen at $-18^{\circ} \mathrm{C}$ until lipid analysis which was done after the last mouse had died. Lipids from both hearts and livers were then extracted from the samples as outlined in Valencak et al. (2003). Phospholipid extracts were transesterified under nitrogen, extracted into hexane, and analysed by GLC (Perkin Elmer Autosystem XL with autosampler and FID, Norwalk, USA) using a capillary column (HP INNOWax, $30 \mathrm{~m} \times 0.25 \mathrm{~mm}$, Hewlett Packard, USA). Fatty acid methyl esters were identified by comparing retention times with those of standards (SigmaAldrich, St. Louis, USA) and peaks were integrated using the Turbochrom 4.1 Software (Perkin Elmer, Norwalk, USA). For all tissue samples, we determined the proportions of the following phospholipid fatty acids: C14:0, C15:0, C16:0, C17:0, C18:0 (saturated fatty acids, SFA), C16:1n-7, C18:1n-9 (monounsaturated fatty acids, MUFA), C18:2n-6, C18:3n-3, C20:4n-6, C20:5n-3, C22:5n-3, and C22:6n-3 (PUFA).

\section{Statistical analyses}

All statistical analyses were carried out using R for Windows (2.9.0.; R Development core Team 2009). To identify important relationships between certain fatty acids and fatty acid classes we performed a principle component analysis which was then followed by ANOVA procedures with the principle component scores as response variables. To account for increased probability of Type I error arising from multiple statistical comparisons, we adjusted significance levels reported in Tables 2 and 3 using the Benjamini-Hochberg method (Benjamini and Hochberg 1995). Body weight data were analysed with a repeated measures design as data were sampled from the same animals throughout the study. This was done by fitting linear mixed effect models with separate intercepts for each female included as the random factor, using the R-package nlme (Pinheiro et al. 2008). Comparisons between feeding regimes were analysed using ANCOVA with body weight entered as covariate. The influence of diet on life span of the animals was tested using (1) ANCOVA and (2) a log 
Table 2 Fatty acid composition of liver phospholipids of C57BL/6 mice maintained on three different diets
Values are given in wt\%, mean \pm SEM. The strength of feeding related membrane composition changes is indicated by $F$ statistics and corresponding $P$ values (Benjamini-Hochberg corrected)

Table 3 Fatty acid composition of heart phospholipids of C57BL/6 mice maintained on three different diets
Values are given in wt\%, mean \pm SEM. The strength of feeding related membrane composition changes is indicated by $F$ statistics and corresponding $P$ values (Benjamini-Hochberg corrected)

\begin{tabular}{lccccl}
\hline & $\begin{array}{l}\mathrm{n}-3 \text { rich } \\
(n=14)\end{array}$ & $\begin{array}{l}\mathrm{n}-6 \text { rich } \\
(n=11)\end{array}$ & $\begin{array}{l}\text { Control } \\
(n=12)\end{array}$ & $F$ & $P$ \\
\hline C14:0 & $0.55 \pm 0.15$ & $1.12 \pm 0.35$ & $0.73 \pm 0.16$ & 1.54 & 0.24 \\
C15:0 & $0.18 \pm 0.02$ & $0.27 \pm 0.08$ & $0.37 \pm 0.06$ & 1.55 & 0.24 \\
C16:0 & $18.9 \pm 0.06$ & $21.8 \pm 1.6$ & $20.87 \pm 0.8$ & 2.2 & 0.16 \\
C17:0 & $0.6 \pm 0.02$ & $0.5 \pm 0.02$ & $0.41 \pm 0.02$ & 15.8 & $\mathbf{0 . 0 0 0 2}$ \\
C18:0 & $19.8 \pm 0.7$ & $20.6 \pm 0.7$ & $19.4 \pm 0.5$ & 1.1 & 0.351 \\
C16:1n-7 & $0.4 \pm 0.05$ & $0.2 \pm 0.05$ & $0.6 \pm 0.05$ & 14.8 & $\mathbf{0 . 0 0 0 2}$ \\
C18:1n-9 & $6.2 \pm 0.5$ & $7.98 \pm 0.6$ & $8.04 \pm 0.6$ & 2.9 & 0.105 \\
C18:2n-6 & $10.9 \pm 0.5$ & $15.5 \pm 0.7$ & $10.8 \pm 0.5$ & 19.97 & $\mathbf{0 . 0 0 0 2}$ \\
C18:3n-3 & $0.7 \pm 0.008$ & $0.26 \pm 0.005$ & $0.05 \pm 0.001$ & 11.6 & $\mathbf{0 . 0 0 0 2}$ \\
C20:4n-6 & $9.9 \pm 1.4$ & $20.7 \pm 1.4$ & $23.2 \pm 0.9$ & 24.8 & $\mathbf{0 . 0 0 0 2}$ \\
C20:5n-3 & $5.3 \pm 0.8$ & $0.21 \pm 0.07$ & $0.12 \pm 0.02$ & 27.9 & $\mathbf{0 . 0 0 0 2}$ \\
C22:5n-3 & $1.84 \pm 0.24$ & $0.39 \pm 0.04$ & $0.66 \pm 0.08$ & 21.2 & $\mathbf{0 . 0 0 0 2}$ \\
C22:6n-3 & $25.9 \pm 1.5$ & $11.83 \pm 1.41$ & $15.5 \pm 0.83$ & 22.23 & $\mathbf{0 . 0 0 0 2}$ \\
$\Sigma$ SFA & $39.9 \pm 0.8$ & $44.3 \pm 2.1$ & $41.8 \pm 1.1$ & 2.3 & 0.15 \\
$\Sigma$ MUFA & $6.6 \pm 0.5$ & $8.2 \pm 0.6$ & $8.6 \pm 0.6$ & 2.7 & 0.11 \\
$\Sigma$ PUFA & $53.95 \pm 0.6$ & $48.7 \pm 1.63$ & $50.3 \pm 0.95$ & 6.8 & 0.005 \\
$\Sigma$ N6 & $20.9 \pm 1.8$ & $36.2 \pm 1.4$ & $33.95 \pm 0.8$ & 27.6 & $\mathbf{0 . 0 0 0 2}$ \\
$\Sigma$ N3 & $33.04 \pm 2.0$ & $12.5 \pm 1.4$ & $16.3 \pm 0.8$ & 42.6 & $\mathbf{0 . 0 0 0 2}$ \\
UI & $259.3 \pm 5.5$ & $196.1 \pm 8.95$ & $219.9 \pm 4.9$ & 21.8 & $\mathbf{0 . 0 0 0 2}$ \\
PI & $300.7 \pm 9.2$ & $196.8 \pm 11.9$ & $232.5 \pm 6.5$ & 28.5 & $\mathbf{0 . 0 0 0 2}$ \\
\hline & & & & &
\end{tabular}

\begin{tabular}{lccccl}
\hline & $\begin{array}{l}\mathrm{n}-3 \text { rich } \\
(n=14)\end{array}$ & $\begin{array}{l}\mathrm{n}-6 \text { rich } \\
(n=11)\end{array}$ & $\begin{array}{l}\text { Control } \\
(n=13)\end{array}$ & $F$ & $P$ \\
\hline C14:0 & $0.58 \pm 0.01$ & $0.6 \pm 0.08$ & $0.7 \pm 0.04$ & 2.1 & 0.17 \\
C15:0 & $1.4 \pm 0.1$ & $2.7 \pm 0.7$ & $2.3 \pm 0.4$ & 2.5 & 0.12 \\
C16:0 & $10.5 \pm 0.2$ & $10.7 \pm 0.4$ & $10.8 \pm 0.2$ & 0.8 & 0.49 \\
C17:0 & $0.4 \pm 0.01$ & $0.3 \pm 0.01$ & $0.3 \pm 0.01$ & 16.8 & $\mathbf{0 . 0 0 0 2}$ \\
C18:0 & $18.5 \pm 0.5$ & $20.2 \pm 1.0$ & $19.9 \pm 0.4$ & 1.23 & 0.35 \\
C16:1n-7 & $0.47 \pm 0.04$ & $0.1 \pm 0.02$ & $0.3 \pm 0.02$ & 34.2 & $\mathbf{0 . 0 0 0 2}$ \\
C18:1n-9 & $5.4 \pm 0.2$ & $4.97 \pm 0.2$ & $6.2 \pm 0.2$ & 7.3 & $\mathbf{0 . 0 0 3}$ \\
C18:2n-6 & $11.6 \pm 1.2$ & $25.4 \pm 1.2$ & $18.5 \pm 0.6$ & 40.6 & $\mathbf{0 . 0 0 0 2}$ \\
C18:3n-3 & $0.1 \pm 0.01$ & $0.12 \pm 0.05$ & $0.08 \pm 0.01$ & 0.4 & 0.7 \\
C20:4n-6 & $5.1 \pm 0.6$ & $11.5 \pm 0.8$ & $11.02 \pm 0.5$ & 26.9 & $\mathbf{0 . 0 0 0 2}$ \\
C20:5n-3 & $0.8 \pm 0.1$ & $0.2 \pm 0.02$ & $0.01 \pm 0.02$ & 33.6 & $\mathbf{0 . 0 0 0 2}$ \\
C22:5n-3 & $2.2 \pm 0.1$ & $0.64 \pm 0.06$ & $0.9 \pm 0.7$ & 57.2 & $\mathbf{0 . 0 0 0 2}$ \\
C22:6n-3 & $43.5 \pm 1.8$ & $23.2 \pm 1.6$ & $29.6 \pm 0.6$ & 45.4 & $\mathbf{0 . 0 0 0 2}$ \\
$\Sigma$ SFA & $31.3 \pm 0.8$ & $34.4 \pm 0.6$ & $33.95 \pm 0.4$ & 7.0 & $\mathbf{0 . 0 0 2}$ \\
$\Sigma$ MUFA & $5.9 \pm 0.3$ & $5.1 \pm 0.2$ & $6.5 \pm 0.2$ & 8.3 & $\mathbf{0 . 0 0 2}$ \\
$\Sigma$ PUFA & $60.4 \pm 0.7$ & $61.1 \pm 0.4$ & $60.2 \pm 0.6$ & 6.5 & $\mathbf{0 . 0 0 6}$ \\
$\Sigma$ N6 & $16.7 \pm 1.8$ & $36.96 \pm 1.5$ & $29.6 \pm 0.5$ & 49 & $\mathbf{0 . 0 0 0 2}$ \\
$\Sigma$ N3 & $46.7 \pm 2.02$ & $24.2 \pm 1.6$ & $30.7 \pm 0.5$ & 48.8 & $\mathbf{0 . 0 0 0 2}$ \\
UI & $326.2 \pm 7.5$ & $245.9 \pm 5.4$ & $270.5 \pm 2.7$ & 45.1 & $\mathbf{0 . 0 0 0 2}$ \\
PI & $398.8 \pm 12.4$ & $262.7 \pm 9.04$ & $305.7 \pm 3.7$ & 48.4 & $\mathbf{0 . 0 0 0 2}$ \\
\hline & & & & &
\end{tabular}

rank test (function "survdiff" in the R library "survival" (Therneau and Lumley 2009), R package version 2.7.2, http://CRAN.R-project.org/package "survival”).
For all samples, an unsaturation index (UI) as well as a peroxidisability index (PI) was computed. The UI represents the number of double bonds per 100 fatty acids 
(Couture and Hulbert 1995) whereas the PI indicates the risk of peroxidisability (Pamplona et al. 1998). These were calculated using the following two relations: UI = $[(\%$ Monoenoic $\times 1)+(\%$ Dienoic $\times 2)+(\%$ Trienoic $\times 3)$ $+(\%$ Tetraenoic $\times 4)+(\%$ Pentaenoic $\times 5)+(\%$ Hexaenoic $\times 6)]$; PI $=(\%$ Monoenoic $\times 0.025)+(\%$ Dienoic $\times$ $1)+(\%$ Trienoic $\times 2)+(\%$ Tetraenoic $\times 4)+(\%$ Pentaenoic $\times 6)+(\%$ Hexaenoic $\times 8)]$.

\section{Results}

Body weight

Body weights were analysed with repeated measurements ANOVA and were significantly lower in the control group than in the n-3 and n-6 enriched groups $\left(F_{2,37}=3.3\right.$, $P=0.047$, Fig. 1). Body weights of individuals decreased over the last half of their life $\operatorname{span}\left(F_{1,2385}=49.75\right.$, $P<0.0001$, Fig. 1). However, this effect was not equal across groups, but rather, was more pronounced in the n-3 and n-6 rich groups (interaction: age $\times$ group, $\left.F_{2,2385}=9.15 ; P<0.0001\right)$. Mean body weights in all three groups (over the entire experiment) and SEMs were $30.9 \pm 0.1,29.9 \pm 0.1$ and $28.7 \pm 0.09$ for $n-3$ rich, $n-6$ rich and controls, respectively. No relationship was found between life span and body weight of individuals across groups $\left(F_{1,34}=2.06 ; P=0.16\right)$.

\section{Liver phospholipid composition}

Dietary supplementation of either n-3 or n-6 PUFAs determined the major fatty acid components of liver phospholipids (Fig. 2a; Table 2). The fatty acid with the highest percentage in the n-3 rich group was C22:6n-3 (DHA) with $25.9 \%$ followed by C18:0 and C16:0 with 19.8 and $18.9 \%$, respectively. Interestingly, the three predominant fatty acids in the n- 6 rich group were C16:0, C20:4n-6 and finally, C18:2n-6. In the control group the three major fatty acid membrane constituents were identified as C16:0, C20:4 and C18:0.

Among single fatty acids, the proportions of 7 out of 13 fatty acids differed significantly between the experimental groups (Table 2). SFAs were found in equal proportions in liver phospholipids from all groups. PUFAs were highest in the $n-3$ rich experimental group, mostly at the expense of MUFAs (Table 2).

The first two principle components (PCs) accounted for nearly $86 \%$ of the variance between individuals. Firstly, individuals differed in the ratio between n-6 and n-3 PUFAs (PC1) which explained $71.5 \%$ of the total variance. The n-3 PUFA content in liver phospholipids differed significantly between treatment groups $\left(F_{1,32}=41.2\right.$;
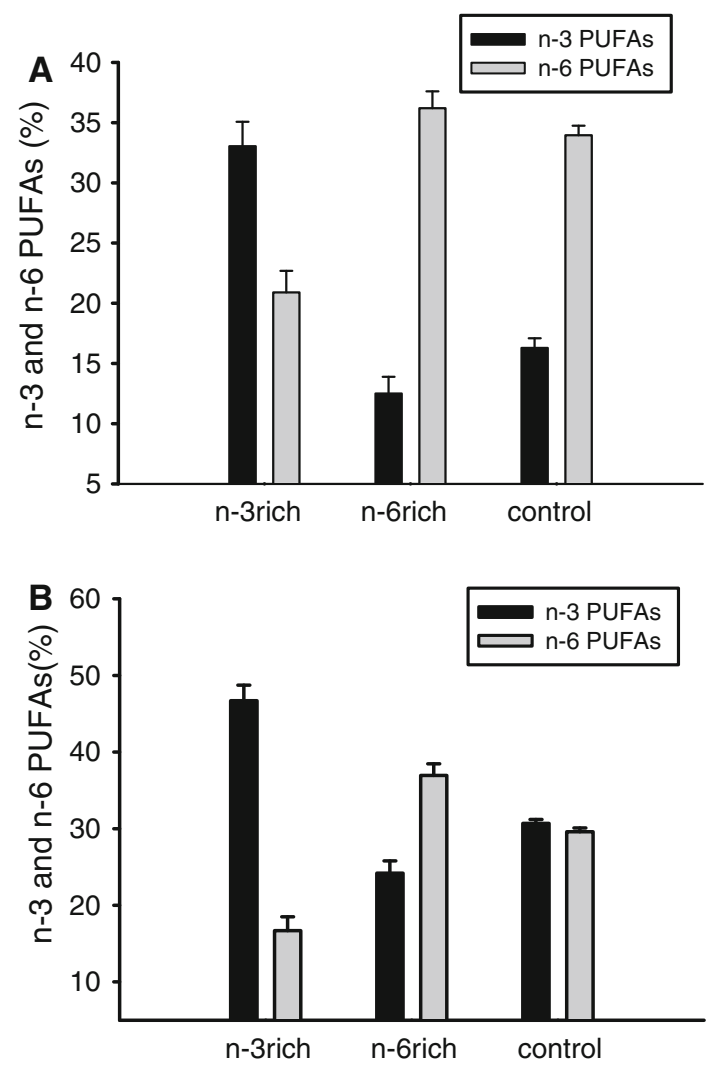

Fig. 2 Distribution of n-3 and n-6 PUFAs (\%) in liver phospholipids (a) and heart phospholipids (b) from animals maintained on three experimental diets. Mean \pm SEM. Sample sizes are indicated in Tables 2 and 3

$P<0.0001)$ as did n-6 PUFA content $\left(F_{1,23}=25.5\right.$; $P<0.0001)$. Secondly, mice with both low SFAs and low levels of linoleic acid (C18:2n-6) had high levels of arachidonic acid (C20:4n-6) and DHA (C22:6n-3) and vice versa (PC2). PC2 explained another $14.5 \%$ of the total variance. Together, our experiment showed that n-3 PUFA contents in mouse liver phospholipids were more than doubled in the $n-3$ rich group compared to the control group (33.04 vs. 16.3\%). As for n-6 PUFA content the differences were not equally pronounced $(36 \%$ in the $n-6$ rich group vs. $34 \%$ in the control group). Interestingly, both n-3 and n-6 PUFA content in liver phospholipids were significantly affected by body weight (n-3: $F_{1,35}=4.9$, $P=0.03$; n-6: $\left.F_{1,35}=4.6, P=0.04\right)$ but SFA, MUFA and PUFA proportions were not. n-3 PUFA content increased with increasing body weight (particularly in the n-3 rich group), whereas n-6 PUFA content was lower in mice with higher body weights. A model computed with the most important fatty acid classes as dependent variable and both experimental feeding group and life span as factors again revealed a large impact of diet on liver membrane composition, i.e., SFA, MUFA, PUFA content (Table 2). 
The UI computed for liver phospholipids differed between the groups $\left(F_{2,33}=21.8, P=0.0002\right.$, Table 2) and was highest in the $n-3$ group. The PI again was highest in the n-3 rich group $\left(F_{2,33}=28.5, P=0.0002\right.$; Table 2$)$.

Heart phospholipid composition

Overall PUFA content in heart phospholipids was approximately $10 \%$ higher than in liver phospholipids and this effect was explained by a $10 \%$ smaller SFA proportion in heart membranes (Table 3). Correspondingly, heart MUFA content did not deviate much from liver membranes (Tables 2, 3). Again, dietary supplementation of either n-3 or n-6 PUFAs had a strong impact on heart phospholipid composition, especially on the $n-3 / n-6$ distribution (Fig. 2b; Table 2). Correspondingly, the predominant membrane constituents were C22:6n-3, C18:2n-6 and C22:6n-3 for the n-3 rich, n-6 rich and the control group, respectively. In heart phospholipids the proportions of eight single fatty acids differed significantly between experimental groups (Table 3).

In heart phospholipid data, $\mathrm{PC} 1$ reflecting the ratio between n-6 PUFAs (plus C18:0) and C22:6n-3 explained $90 \%$ of the total variance. Interestingly, the n-3 PUFA class was most enriched in heart phospholipids compared to liver phospholipids. n-6 PUFAs at most amounted to about $36 \%$ in total. The UI was again highest in the n-3 rich group and differed significantly from the other feeding groups $\left(F_{2,34}=45.1, P=0.0002\right.$, Table 3$)$. Similarly, the PI differed between the groups and was highest in the n-3 rich group $\left(F_{2,34}=48.4, P=0.0002\right.$, Table 2$)$.

\section{Life span}

As illustrated in Figs. 3 and 4, our experimental manipulation did not influence age at death in our animals $\left(F_{2,37}=0.98, P=0.39\right)$. Mean age at death in all three

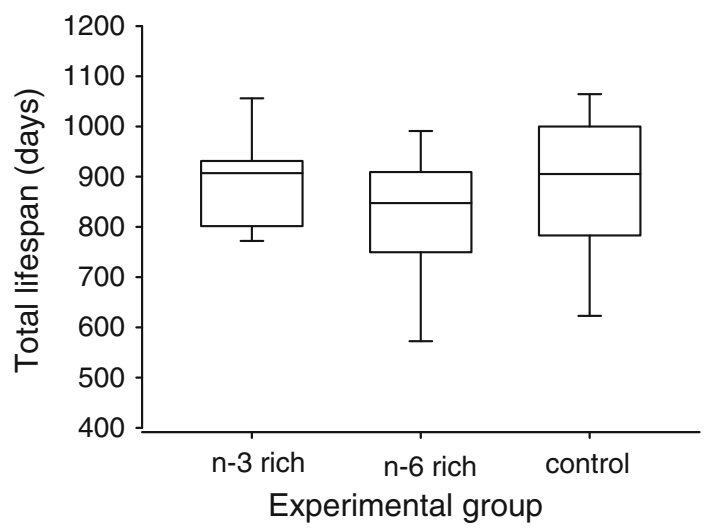

Fig. 3 Boxplot of median lifespan in all three experimental groups, $N=40$

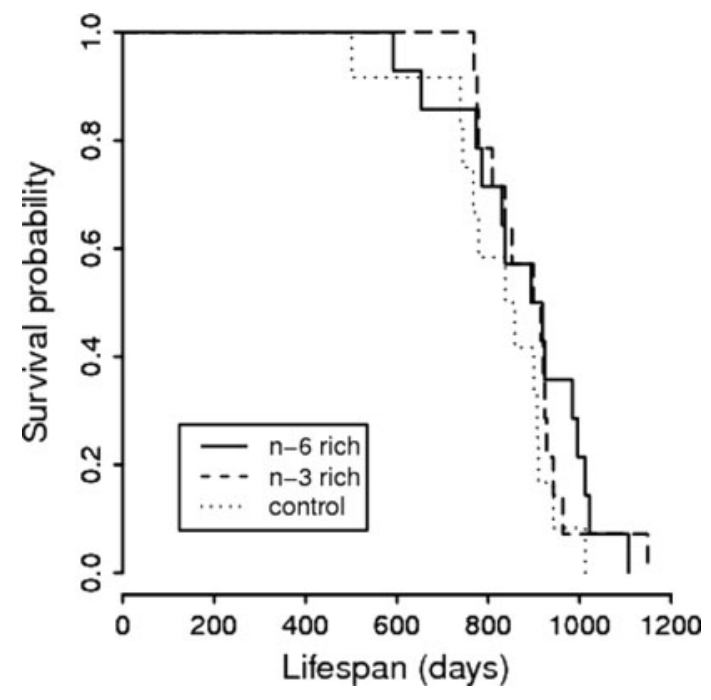

Fig. 4 Age specific mortality in animals from all three dietary regimes, $N=40$

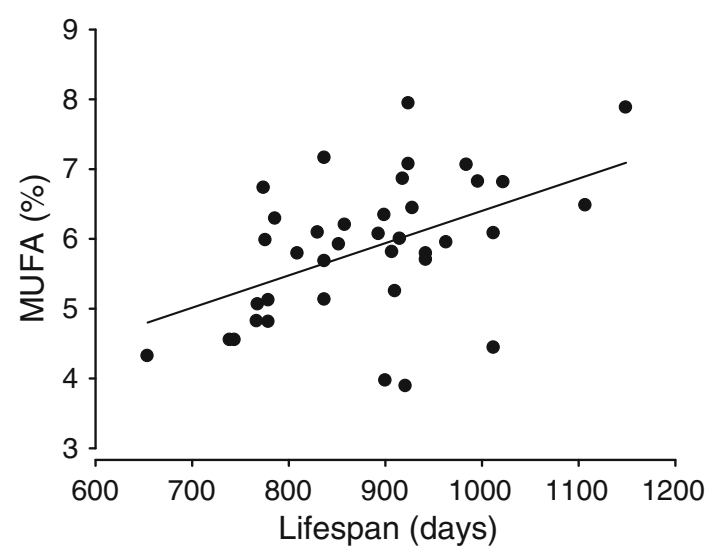

Fig. 5 Relationship between heart phospholipid monounsaturated fatty acid (MUFA) content and maximum lifespan (MLSP) in animals from all groups $(N=40)$

groups was $867.2 \pm 20.1$ days. Across groups mean age equalled $890.1 \pm 27,824.7 \pm 38,880.9 \pm 39$ days for $n-3$ rich, n-6 rich, and control groups, respectively. Log-rank testing confirmed the lack of significant differences in life span in n-3 rich, n-6 rich and control animals $\left(\chi^{2}=2.3\right.$; $P=0.319)$. Median life span was 896 days for all three groups with lower and upper confidence limits of 837 and 924 days, respectively.

Multivariate statistical models computed with the most important fatty acid classes as dependent variables and life span and feeding group as the independent variable revealed a significant positive relationship between heart phospholipid MUFA content and life $\operatorname{span}\left(F_{1,32}=15.05\right.$, $P=0.0005$, Fig. 5). This relationship was absent, however, in liver phospholipids. No interaction was found between life span and feeding group $\left(F_{2,32}=0.844\right.$, $P=0.44)$. 


\section{Discussion}

Body weight and membrane composition

Due to the fact that diets were not isocaloric (Table 1), it was not surprising that mice on the enriched diets had higher body weights than animals on the control diet and possibly have built up fat reserves. A slightly higher energy content of the diet in the $n-3$ rich group (Table 1) may also explain why the highest body weights were found in this group (Fig. 1). In agreement with our primary expectation, dietary n-3 and n-6 fatty acid supplementation significantly affected heart and liver phospholipid membrane composition. Essentially, membrane PUFAs of mice given fatenriched diets reflected the PUFA composition in the diet. The absolute degree of unsaturation as well as the PI was markedly higher in heart membranes than in liver phospholipids. This accords with data from European hares whose heart phospholipids are more unsaturated than those of their liver (Valencak et al. 2003). Our 10\% enrichment of both n-3 and n-6 fatty acids induced a significant increase in membrane unsaturation in both examined tissues, with a PUFA content of almost $54 \%$ in liver and $61 \%$ in heart phospholipids. To our knowledge, these results show the highest level of membrane unsaturation reported for C57BL/6 mice. Hulbert et al. (2006b) already compared tissue composition between the very long-lived naked mole rats and $\mathrm{C} 57 \mathrm{BL} / 6$ mice. While membrane composition of our control group clearly is comparable with data presented in Hulbert et al. (2006b), the PUFA content in both enriched groups was somewhat higher in our study $(+1 \%$ in $n-$ 6 rich, $+7 \%$ in $n-3$ rich liver phospholipids). Note that Hulbert et al. (2006b) did not involve any dietary supplementation of fatty acids and the mice were killed at 3-4 months of age. In our present study, however, animals were allowed to die of natural causes and their mean age at the time of tissue sampling was approximately 29 months. Interestingly, heart phospholipid PUFA content did not differ as much as liver phospholipid PUFA content between the groups (Tables 2, 3). Thus, we conclude that heart tissue composition in laboratory mice is more tightly regulated than liver composition in the same species. Clearly, membrane PUFA content is a regulated trait in mammals in general (Valencak et al. 2003; Valencak and Ruf 2007; Hulbert et al. 2007; Ruf and Arnold 2008). Although, given that the $6 \%$ higher PUFA content in liver tissues in our experimental mice compared to the control group, we confirm other studies which have also shown that depending on the tissue type, PUFAs can readily be incorporated into the tissue if $n-3$ or $n-6$ PUFAs are available in excess. To date, little is known of the mechanisms that regulate membrane fatty acid composition, both within and between species (Hulbert et al. 2007). It has been suggested that membrane fatty acid composition is influenced via a constant membrane remodelling processes (Sprecher 2000; Abbott et al. 2010). During these processes, membrane constituents like fatty acids are constantly removed and replaced through the actions of enzyme groups such as acetyltransferases and also phospholipases, desaturases, and elongases. Because acetyltransferases are highly sensitive for PUFAs but do not discriminate well between n-6 and n-3 PUFAs (Lands et al. 1982; Abbott et al. 2010), membrane n-3 and n-6 composition in our study animals differed strongly (Fig. 2; Tables 2, 3) while overall PUFA content was not so dissimilar. In both tissues, the absolute highest accumulation of dietary fatty acids in the membrane was found in the $n-3$ rich group: in heart phospholipids of $n-3$ fed mice the amount of n-3 PUFAs exceeded $46 \%$ and thus was about $16 \%$ higher than in the control group. Specifically, the amount of DHA in the heart tissues was increased by $14 \%$ compared to control animals and $20 \%$ higher than in mice on the $n-6$ rich diet. We conclude that, if animals are given a diet rich in one certain PUFA class, the tight regulation of membrane fatty acid composition in mammalian membranes is apparently limited to the classes SFA, MUFA, and PUFA. In a more natural situation, however, with animals on non-supplemented diets, it has been shown that total and membrane n-6/n-3 ratios are up-regulated in short or decreasing photoperiod (Geiser et al. 2007; Ruf and Arnold 2008). This is thought to prepare the animals for maintaining functionality of the heart at low tissue temperatures during torpor and hibernation (Ruf and Arnold 2008).

\section{Life span}

Our experimental procedure involved diet-induced membrane composition changes and determination of life span of the animals as suggested by Hulbert et al. (2006a). However, life span clearly was unaffected by this manipulation (Fig. 3), despite the fact that we detected pronounced differences in membrane composition in the mice and thus, might have affected ROS production by the incorporation of certain PUFAs to the tissue. Thus, our results contradict the 'membrane pacemaker hypothesis of aging'. Some possible explanations for this outcome are:

(1) The dietary fatty acid supplementation might not have been strong enough to bring about an increase in lipid peroxidation and ROS production. However, this possibility is very unlikely because a $20 \%$ difference in $n-3$ PUFA content in heart phospholipids, as observed in our study, should have affected lipid peroxidation and ROS production. During lipid peroxidation, a free radical (originating from metabolism) attacks a hydrogen from a polyunsaturated lipid molecule, thereby leaving the 
carbohydrate chain with an unpaired electron and modifying it to a radical itself (Holman 1954). Thus, ROS attack on membrane bilayers should not only harm the molecule and prevent it from proper functioning but even produce more oxidative stress in the end (reviewed in Hulbert 2010). In line with this, even a small difference in membrane PUFA content or n-3 content should greatly affect ROS production. Among small mammals, a $20 \%$ difference in n-3 PUFA content was reported in muscle phospholipids from a mole (Talpa europaea) and a hedgehog (Erinacaeus europaeus) with their maximum life span differing by 10 years (Valencak and Ruf 2007). Also, in support of the 'membrane pacemaker hypothesis of aging', very low n-3 PUFA contents were also reported from erythrocyte membranes in humans (Puca et al. 2008) who clearly are exceptionally long-lived among mammals. Equally, African elephants that live up to 70 years (Carey and Judge 2000) were found to have very low n-3 PUFA contents of 9.6\% in muscle phospholipids (Valencak and Ruf 2007) as opposed to $30 \%$ in heart phospholipids of C57B1/6 mice (this study). Our results suggest that while n-3 PUFA content (and specifically, a high n-3/n-6 PUFA ratio) in muscle phospholipids in a variety of mammals is correlated with a shorter life span (Valencak and Ruf 2007), the same relationship does not hold true within a species. This lack of a causal relation between membrane composition and life span is even more surprising given the fact that we reached the highest reported membrane unsaturation for C57B1/6 mice.

(2) One could argue that we did not confirm the $n-3 / n-6$ PUFA relationship with longevity because we sampled heart and liver tissues instead of skeletal muscle. Skeletal muscle and heart phospholipid fatty acid composition, however, usually correlate very well (Valencak et al. 2003; Hulbert et al. 2007; Valencak and Ruf 2007) so this point seems unlikely. Also, PUFA contents of muscle, heart, and brain co-vary among the longest living rodents and similarly sized, but short-lived mice (Hulbert et al. 2006b) so the sampling of skeletal muscle most likely would not have changed any of our results. As primary sites of ROS generation, mitochondrial membrane fatty acid composition might be of great importance for testing the predictions of the membrane pacemaker hypothesis. Yet, there is evidence available that mitochondrial membrane fatty acid content tightly co-varies with tissue fatty acid composition (reviewed in Hulbert et al. 2007), so we are confident that observing mitochondrial membrane composition would not have changed our conclusions.

(3) Rather, we suggest that the relationship between membrane unsaturation, ROS production, and life span has been overestimated in the past and is in fact too weak to cause a significantly shorter life span. Our results indicate that a high degree of membrane unsaturation, and hence a high potential for ROS production, did not affect life span. On a more general ground, the impact of cellular oxidative damage/ROS on an animal's life span needs further clarification. For instance, naked mole rats that are exceptionally long-lived animals for their size (Buffenstein 2008) possess low membrane unsaturation but at the same time produce high levels of ROS (Andziak et al. 2006; Andziak and Buffenstein 2006). Additionally, orally administered antioxidants have been proven to be ineffective in diminishing ROS (Selman et al. 2006) and finally, over-expression of antioxidant enzymes in mice does not extend life span (Perez et al. 2009). In sum, there is increasing evidence that is inconsistent with the oxidative stress framework of aging thus suggesting that no single component can convincingly explain the complex interactions among factors involved in the aging process. Related to this, we conclude that an experimentally altered membrane composition alone does not dictate life span but rather, tissue membrane composition and MLSP might be linked in a more complex interaction.

\section{Correlation between MUFAs and life span}

Surprisingly, we observed that the content of monounsaturated fatty acids in heart phospholipids was positively related to life span, no matter to which experimental group the animals had been assigned (Fig. 5). The eldest mice had the highest content of monounsaturated fatty acids in their heart phospholipids. High contents of MUFAs have also been reported from liver and skeletal muscle phospholipids in the long-lived echidna (Hulbert et al. 2008). Similarly, Buttemer et al. 2008 showed that cardiac phospholipids of long-living petrels contain about $20 \%$ higher MUFAs than those from shorter living gallinaceous species. While allometric scaling for MUFA contents in skeletal muscle membranes of nine different bird species also suggested that longer living birds have higher MUFAs (Hulbert et al. 2002), the same trend could not be confirmed in a 42-mammal comparison have been found in mammals (Valencak and Ruf 2007). In a feeding trial with similar experimental groups like in this study, Du et al. (2001) did not find any relationship between MUFA content in hepatic triacylglycerols and life span even though they examined the same strain of mice. From epidemiological studies and feeding trials in humans, it has been suggested that MUFAs could positively impact on blood pressure, although the mechanism is yet unknown (Alonso et al. 2006). As mammals can synthesise monounsaturated fatty acids de novo we conclude that, when our experimental animals became older, they may have relocated MUFAs into heart phospholipids. The observed pattern was absent in liver tissues, however, so we need to be cautious with more interpretations of this finding. Further studies are 
needed to identify a causative effect of monosaturated fatty acids on life span.

Acknowledgments During the study, T.G.V. was funded by grant P17794-B06 to T.R. and by grant T 376-B17 to T.G.V. from the Austrian Science Fund, and by the province of Lower Austria. We are grateful to the animal house facility staff (Michaela Salaba, Peter Steiger), and to Beatrice Grafl for their assistance. Barbara Bauer helped with tissue sampling and Katharina Brugger assisted T.G.V. with $\mathrm{R}$ programming. We would like to thank Michael Hämmerle who performed all chemical analyses under direction of Frieda Tataruch. All experiments described here were approved by the ethics committee of the University of Veterinary Medicine Vienna and comply with the current laws in Austria, where the experiments were performed.

Open Access This article is distributed under the terms of the Creative Commons Attribution Noncommercial License which permits any noncommercial use, distribution, and reproduction in any medium, provided the original author(s) and source are credited.

\section{References}

Abbott SK, Else PL, Hulbert AJ (2010) Membrane fatty acid composition of rat skeletal muscle is most responsive the balance of dietary n-3 and n-6 PUFA. Br J Nutr 103:522-529. doi: 10.1017/S0007114509992133

Alonso A, Ruiz-Gutierrez V, Martinez-Gonzalez A (2006) Monounsaturated fatty acids, olive oil and blood pressure: epidemiological, clinical and experimental evidence. PHN 9:251-257. doi: 10.1079/PHN2005836

Andziak B, Buffenstein R (2006) Disparate patterns of age-related changes in lipid peroxidation in long-lived naked mole-rats and shorter-lived mice. Aging Cell 5:525-532. doi:10.1111/j.14749726.2006.00246.x

Andziak B, O'Connor TP, Qi WB, DeWaal EM, Pierce A, Chaudhuri AR, Van Remmen H, Buffenstein R (2006) High oxidative damage levels in the longest-living rodent, the naked mole-rat. Aging Cell 5:463-471. doi:10.1111/j.1474-9726.2006.00237.x

Barja G (2004) Free radicals and aging. Trends Neurosci 27:595-600

Benjamini Y, Hochberg Y (1995) Controlling the false discovery rate: a practical and powerful approach to multiple testing. J Roy Stat Soc B 57:289-300

Brand MD (2000) Uncoupling to survive? The role of mitochondrial inefficiency in ageing. Exp Gerontol 35:811-820

Buffenstein R (2008) Negligible senescence in the longest-living rodent, the naked mole-rat: insights from a successfully aging species. J Comp Physiol B 178:439-445. doi:10.1007/s00360007-0237-5

Buttemer WA, Battam H, Hulbert AJ (2008) Fowl play and the price of petrel: long-living Procellariiformes have peroxidation-resistant membrane composition compared with short-living Galliformes. Biol Lett 4:351-354. doi:10.1098/rsbl.2008.0145

Carey JR, Judge DS (2000) Longevity records: life spans of mammals, birds, reptiles, amphibians and fish. Odense University Press, Denmark

Couture P, Hulbert AJ (1995) Membrane fatty acid composition of tissues is related to body mass of mammals. J Membr Biol 148:27-39

Du C, Sato A, Watanabe S, Ikemoto A, Fujii Y, Okuyama H (2001) Effect of dietary oils enriched with n-3 fatty acids on survival of mice. J Nutr Biochem 12:474-480
Geiser F, McAllan BM, Kenagy GJ, Hiebert SM (2007) Photoperiod affects daily torpor and tissue fatty acid composition in deer mice. Naturwissenschaften 94:319-325. doi:10.1007/s00114006-0193-Z

Holman RT (1954) Autoxidation of fats and related substances. In: Holman RT, Lundberg WO, Malkin T (eds) Progress in chemistry of fats and other lipids, vol 2. Pergamon Press, London, pp 51-98

Hulbert AJ (2010) Metabolism and longevity: is there a role for membrane fatty acids? Integr Comp Biol 50:1-10. doi:10.1093/ icb/icq007

Hulbert AJ, Faulks SC, Buttemer WA, Else PL (2002) Acyl composition of muscle membranes varies with body size in birds. J Exp Biol 205:3561-3569

Hulbert AJ, Faulks SC, Harper JM, Miller RA, Buffenstein R (2006a) Extended longevity of wild-derived mice is associated with peroxidation-resistant membranes. Mech Aging Dev 127:653657. doi:10.1016/j.mad.2006.03.002

Hulbert AJ, Faulks SC, Buffenstein R (2006b) Oxidation-resistant membrane phospholipids can explain longevity differences among the longest-living rodents and similar-sized mice. J Gerontol A 61:1009-1018

Hulbert AJ, Pamplona R, Buffenstein R, Buttemer WA (2007) Life and death: metabolic rate, membrane composition, and life span of animals. Physiol Rev 87:1175-1213. doi:10.1152/physrev. 00047.2006

Hulbert AJ, Beard LA, Grigg GC (2008) The exceptional longevity of an egg-laying mammal, the short-beaked echidna (Tachyglossus aculeatus) is associated with peroxidation-resistant membrane composition. Exp Gerontol 43:729-733. doi:10.1016/j.exger. 2008.05.015

Lands WE, Inoue M, SugiuraY OkuyamaH (1982) Selective incorporation of polyunsaturated fatty acids into phosphatidylcholine by rat liver microsomes. J Biol Chem 257:14968-14972

Nehring K (1960) Agrikulturchemische Untersuchungsmethoden für Dünge- und Futtermittel. Böden und Milch, Parey

Otzelberger K (1983) Österreichisches Methodenbuch für die Untersuchung von Futtermitteln Futterzusatzstoffen und Schadstoffen Arbeitsgemeinschaft der Landwirtschaftlichen. Versuchsanstalten in Österreich, Wien

Pamplona R, Barja G (2007) Highly resistant macromolecular components and low rate of generation of endogenous damage: two key traits of longevity. Ageing Res Rev 6:189-210. doi: 10.1016/j.arr.2007.06.002

Pamplona R, Portero-Otin M, Riba D, Ruiz C, Prat J, Bellmunt MJ, Barja G (1998) Mitochondrial membrane peroxidizability index is inversely related to maximum lifespan in mammals. J Lipid Res 39:1989-1994

Perez VI, Van Remmen H, Bokov A, Epstein CJ, Vijg J, Richardson A (2009) The overexpression of major antioxidant enzymes does not extend lifespan of mice. Aging Cell 8:73-75. doi: 10.1111/j.1474-9726.2008.00449.x

Pinheiro J, Bates D, DebRoy S, Sarkar D, R Core Team (2008) nlme: linear and nonlinear mixed effects models. $\mathrm{R}$ package version $3: 1-88$

Puca AA, Andrew P, Novelli V, Anselmi CV, Somalvico F, Cirillo NA, Chatgilialoglu C, Ferreri C (2008) Fatty acid profile of erythrocyte membranes as possible biomarker of longevity. Rejuv Res 11:63-72. doi:10.1089/rej.2007.0566

R Development Core Team (2009) R: a language and environment for statistical computing. R Foundation for Statistical Computing, Vienna (http://www.R-project.org)

Ruf T, Arnold W (2008) Effects of polyunsaturated fatty acids on hibernation and torpor: a review and hypothesis. Am J Physiol R 294:1044-1052. doi:10.1152/ajpregu.00688.2007 
Selman C, McLaren JS, Meyer C, Duncan JS, Redman P, Collins AR, Duthie GG, Speakman JR (2006) Life-long vitamin C supplementation in combination with cold exposure does not affect oxidative damage or lifespan in mice, but decreases expression of antioxidant protection genes. Mech Ageing Dev 127:897-904. doi:10.1016/j.mad.2006.09.008

Speakman JR (2005) Review: body size, energy metabolism and lifespan. J Exp Biol 208:1717-1730. doi:10.1242/jeb.01556

Sprecher H (2000) Metabolism of highly unsaturated n-3 and n-6 fatty acids. Biochim Biophys Acta 1486:219-231

Therneau T, Lumley T (2009) Survival analysis, including penalised likelihood. URL http://r-forge.r-project.org
Turner N, Else PL, Hulbert AJ (2003) Docosahexaenoic acid (DHA) content of membranes determines molecular activity of the sodium pump: implications for disease states and metabolism. Naturwissenschaften 90:521-523

Valencak TG, Ruf T (2007) n-3 polyunsaturated fatty acids impair lifespan but have no role for metabolism. Aging Cell 6:15-25. doi:10.1111/j.1474-9726.2006.00257.x

Valencak TG, Arnold W, Tataruch F, Ruf T (2003) High content of polyunsaturated fatty acids in muscle phospholipids of a fast runner, the European brown hare (Lepus europaeus). J Comp Physiol B 173:695-702 